\section{International Scientific Journal Theoretical \& Applied Science}

p-ISSN: 2308-4944 (print) e-ISSN: 2409-0085 (online)

Year: $2014 \quad$ Issue: $10 \quad$ Volume: 18

Published: $30.10 .2014 \quad$ http://www.T-Science.org
Anatoly Aleksandrovich Naumov Docent, Candidate of Technical Sciences, Center of Applied Mathematical Research,

Novosibirsk, Russia,

a_a_naumov@mail.ru

Anastasia Anatolievna Naumova

Marketing Director, MBA, Marie Forleo Business School, NYC,

Manchester, UK, anastasia.naumova@yahoo.com

SECTION 31. Economic research, finance, innovation.

\title{
TO GENERATING OF FINANCIAL FLOWS IN PROBLEMS OF ANALYSIS ON BUSINESS EFFICIENCY
}

Abstract: The paper reports the results of a study of methods of detailing of financial flows. The advantages of using these methods for the estimating of business effectiveness are shown. The calculating schemes for income and yield obtained.

Key words: Capital structure, borrowing, optimization, modeling, financial flows, detailing flows method.

Language: Russian

Citation: Naumov AA, Naumova AA (2014) TO GENERATING OF FINANCIAL FLOWS IN PROBLEMS OF ANALYSIS ON BUSINESS EFFICIENCY. ISJ Theoretical \& Applied Science 10 (18): 53-55. doi: http://dx.doi.org/10.15863/TAS.2014.10.18.13

УДК 336.77: 330.42

\section{К ГЕНЕРАЦИИ ФИНАНСОВЫХ ПОТОКОВ В ЗАДАЧАХ АНАЛИЗА БИЗНЕСА НА ЭФФЕКТИВНОСТЬ}

Аннотация: В работе приведены результаты исследования методов детализации финансовых потоков. Показаны преимущества использования этих методов при оценивании эффективности бизнеса. Получены расчетные схемы для показателей дохода и доходности.

Ключевые слова: Финансовые потоки, метод детализации потоков, моделирование, эффективность, доход, доходность.

Введение. Задача управления финансовыми потоками бизнеса - одна из самых интересных с теоретической и практической точек зрения задач. Суть этой задачи состоит в том, чтобы наилучшим образом в темпе функционирования бизнеса проводить анализ текущего состояния потоков и принимать решение по их изменению (корректировке, управлению). Такие задачи возникают в тех случаях, когда решается вопрос: как наилучшим образом объединить в бизнесе финансовые потоки от нескольких источников финансирования, какие объемы средств и на каких условиях заимствовать, чтобы в сочетании с собственными средствами это позволило бы получить наибольший эффект для бизнеса? Особенностью этих задач является то, что они должны решаться в рамках более общей задачи планирования развития бизнеса вообще.

Принцип генерации финансовых потоков. В основу этого принципа положены простые идеи: основные финансовые потоки бизнеса (вложений и доходов) порождают (генерируют) вспомогательные финансовые потоки (заимствований, расчетов по кредитам, внешнего использования и др.). Заметим, что если основные потоки носят стратегический характер и прописаны в бизнес-плане, то вспомогательные формируются исходя из тактических соображений. Правда, возможно, что вспомогательные потоки могут приводить к изменениям в основных потоках (изменяются условия работы с заказчиками, поставщиками, потребителями и т.д.).

Постановка задачи. Пусть для некоторого бизнеса известны входной и выходной финансовые потоки в виде: $F_{\text {in }}(t), \quad t=$ $t_{0}, t_{1}, t_{2}, \ldots,\left(t_{m}=T\right)$, - входной (инвестиций, затрат, вложений) финансовый поток, $F_{\text {out }}(t), t=$ $t_{0}, t_{1}, t_{2}, \ldots,\left(t_{m}=T\right), \quad-\quad$ выходной $\quad$ (доходов) финансовый поток. Будем предполагать, что моменты времени $t_{0}, t_{1}, t_{2}, \ldots,\left(t_{m}=T\right)$ положительные целые числа и все интервалы 
между соседними отсчетами равны единице времени. Требуется оценить эффективность бизнеса.

Оценивание эффективности бизнеса на основе метода детализации потоков. Будем анализировать последовательно элементы множества $\left\{F_{\text {in }}(t)\right\}, \quad t=t_{0}, t_{1}, t_{2}, \ldots, t_{m}, \quad$ для возрастающих значений моментов времени. Пусть $F_{i n}\left(t_{0}\right)$ - это заемные средства по ставке $r_{i n, 0}$, которые необходимо будет погашать в виде одинаковых выплат в интервале времени $\left[t_{0} ; T_{0}\right]$. Тогда значение выплат по кредиту составит: $F_{i n, R C, 0}=F_{i n}\left(t_{0}\right) \cdot \frac{r_{i n, 0}}{1-\left(1+r_{i n, 0}\right)^{-n_{0}}}$. Таким образом, элемент входного потока $F_{i n}\left(t_{0}\right)$ породил (сгенерировал) поток выплат по кредиту: $F_{i n, D F, 0}=\left(0, F_{i n, R C, 0}, F_{i n, R C, 0}, \ldots F_{i n, R C, 0}\right)-$ вектор из $\left(n_{0}+1\right)$-го элемента, каждый из элементов которого привязан к моментам времени $t_{0}, t_{1}, t_{2}, \ldots, t_{n_{0}}=T_{0}$ соответственно. Продолжаем применять эту процедуру анализа элементов входного потока и генерации элементов потока выплат по кредитам до момента времени $t=t_{m}$ (при этом получим вектор $F_{i n, D F, m}$ ). В результате свертки векторов $F_{i n, D F, i}, i=0,1,2, \ldots, m$, получим новый (сгенерированный, порожденный входным потоком) вектор $F_{i n, D F}^{\Sigma}$.

Переходим к анализу элементов выходного финансового потока бизнеса $F_{\text {out }}(t), t=$ $t_{0}, t_{1}, t_{2}, \ldots, t_{m}$. Основная идея состоит в том, чтобы расписать (распределить) элементы этого потока в два потока: один будет показывать, как компенсируются (погашаются) элементы потока $F_{i n, D F}^{\Sigma}$ (будем обозначать его через $F_{o u t, I P}^{\Sigma}$, где $I P$ Internal Projects) и второй - поток, который может быть выведен из данного бизнеса и использован вне него (будем обозначать этот поток через $F_{o u t, E P}^{\Sigma}$, где EP - External Projects). Как и для элементов входного потока, в данном случае можно записать: $F_{\text {out }, I P}^{\Sigma}=\oplus_{i=0}^{m} F_{\text {out }, I P, i}$ и $F_{\text {out }}=$ $F_{\text {out }, I P}^{\Sigma} \oplus F_{\text {out }, E P}^{\Sigma} . \quad$ Здесь $\quad F_{\text {out }}=$ $\left(F_{\text {out }}\left(t_{0}\right), F_{\text {out }}\left(t_{1}\right), \ldots, F_{\text {out }}\left(t_{m}\right)\right)$. Если равенства $F_{\text {out }, I P}^{\Sigma}(t)=F_{i n, D F}^{\Sigma}(t)$ для всех $t \in\left\{t_{0}, t_{1}, t_{2}, \ldots, t_{m}\right\}$ выполнились, то можно перейти к оцениванию показателя эффективности $N F V_{D F}$ в соответствии с формулой: $\quad N F V_{D F}=\sum_{t} F_{o u t, E P}^{\Sigma}(t) \cdot(1+$ $\left.r_{\text {out }, E P}(t)\right)^{T-t}$. Здесь $F_{\text {out,EP }}^{\Sigma}(t)$ - элемент вектора $F_{o u t, E P}^{\Sigma}$, относящийся к моменту времени $t$, $r_{\text {out }, \text { EP }}(t)$ - ставка внешнего использования средств в размере $F_{o u t, E P}^{\Sigma}(t)$. Заметим, что в общем случае таких ставок может быть несколько и они соответствуют доходностям бизнесов, в которые вкладываются средства $F_{o u t, E P}^{\Sigma}(t)$.

Опираясь на выражение для оценивания дохода $N F V_{D F}$, можно предложить расчетные схемы для оценивания доходности бизнеса, срока его окупаемости и т.д. Например, для оценивания доходности можно воспользоваться формулами: $I R R_{D F, i n, N F V+D F}=$

$\left\{r \mid \sum_{t} F_{\text {in }}(t) \cdot(1+r)^{T-t}=N F V_{D F}+\sum_{t} F_{i n, D F}^{\Sigma}(t)\right\}$, $I R R_{D F, i n}=\left\{r \mid \sum_{t} F_{i n}(t) \cdot(1+r)^{T-t}=N F V_{D F}\right\}$,

$I R R_{D F, D F}=\left\{r \mid \sum_{t} F_{i n, D F}^{\Sigma}(t) \cdot(1+r)^{T-t}=N F V_{D F}\right\}$.

Выводы. Отметим положительные моменты, связанные с использованием метода детализации потоков и оцениванием показателя дохода бизнеса $N F V_{D F}$. 1) Все ставки, используемые при расчете показателя, имеют ясный смысл и выбираются достаточно однозначно (это либо банковские ставки для кредитов, либо ставки по депозитам, либо ставки внешних бизнесов, эффективность которых оценена и т.д.). Если какие-либо из этих ставок точно не известны, то в этом случае следует наряду с самим значением показателя $N F V_{D F}$ оценить для него и риск $\Re_{N F V_{D F}}$. Тогда при анализе бизнеса на эффективность следует оперировать парой доход-риск $\left\langle N F V_{D F}, \Re_{N F V_{D F}}\right\rangle$. 2) Значение показателя $N F V_{D F}$ интерпретируется тоже достаточно просто - это доход от бизнеса, который будет получен на момент времени $t=T$. В связи с этим обстоятельством показатель можно было бы обозначить еще и таким образом $N F V_{D F}(T)$, введя в его обозначение время окончания бизнеса или время, на которое проводится анализ бизнеса на эффективность. 3) Фактически элементы $F_{o u t, E P}^{\Sigma}(t)$ в формуле для показателя $N F V_{D F}$ равны разностям между компонентами детализированных (сгенерированных) векторов выходного и входного финансовых потоков.

\section{References:}

1. Naumov AA (2013) Metodyi analiza i sinteza investitsionnyih proektov. Effektivnost, riski, upravlenie. LAP LAMBERT Academic Publishing, pp. 356.

2. Naumov AA (2013) Optimizatsiya strukturyi zaimstvovaniy i vlozheniy dohodov investitsionnogo proekta. Materials of the ISPC «Advances in techniques \& technologies», 30.10.2013, Milan, Italy. ISJ Theoretical \& Applied Science 10 (6): 133-136. doi: http://dx.doi.org/10.15863/TAS.2013.10.6.20 
3. Naumov AA (2013) Ispolzovanie metoda detalizatsii finansovyih potokov $\mathrm{v}$ zadachah otsenivaniya effektivnosti proektov. Finansovaya analitika: problemyi i resheniya. No. 48 (186), pp. 35-41.

4. Naumov AA (2014) Otsenivanie effektivnosti integrirovannyih proektov. Finansovaya analitika: problemyi i resheniya, No. 8 (194), pp. 36-43.

5. Naumov AA (2014) Analiz kriteriev effektivnosti investitsionnyih proektov. Materials of the ISPC «Modern mathematics in science», 30.06.2014, Caracas, Venezuela. ISJ Theoretical \& Applied Science 6 (14): 92-94. doi:

http://dx.doi.org/10.15863/TAS.2014.06.14.19

6. Naumov AA, Naumova AA (2014) O nekorrektnosti odnoy modeli optimizatsii strukturyi kapitala. Materials of the ISPC «European Innovation», 30.09.2014, Martigues, France. ISJ Theoretical \& Applied Science 9 (17): 170-173. doi: http://dx.doi.org/10.15863/TAS.2014.09.17.29
7. Naumov AA (2013) Modifikatsiya kriteriya NFV na osnove metoda detalizatsii finansovyih potokov proektov. Materials of the ISPC «Results \&Perspectives», 30.09.2013, Florence, Italy. ISJ Theoretical \& Applied Science 9 (5): 98-102.

doi: http://dx.doi.org/10.15863/TAS.2013.09.5.12

8. Ostroushko AA, Bazhenov RI (2014) Analiz assortimenta elektrotovarov $\mathrm{s}$ ispolzovaniem ABC-analiza. Ekonomika i menedzhment innovatsionnyih tehnologiy, No. 10 [Elektronnyiy resurs]. URL: http://ekonomika.snauka.ru/2014/10/6033 (data obrascheniya: 08.10.2014).

9. Bazhenov RI, Veksler VA, Grinkrug LS (2014) RFM-analiz klientskoy bazyi $\mathrm{v}$ prikladnom reshenii 1S:Predpriyatie 8.3. Informatizatsiya i svyaz, No. 2, pp. 51-54.

10. Spisok trudov [Elektronnyiy resurs]. URL: https://sites.google.com/site/anatolynaumov201 1/home/spisok-trudov-list-of-papers (data obrascheniya: 27.10.2014). 